\title{
AOA, Delay, and Complex Propagation Factor Estimation for the Monostatic MIMO Radar System
}

\author{
Saleh 0. Al-Jazzar (D) and Sami Aldalahmeh \\ Al-Zaytoonah University of Jordan, Amman, Jordan \\ Correspondence should be addressed to Saleh O. Al-Jazzar; saleh.g@zuj.edu.jo
}

Received 19 June 2018; Revised 29 August 2018; Accepted 12 September 2018; Published 22 November 2018

Academic Editor: Giorgio Montisci

Copyright ( 2018 Saleh O. Al-Jazzar and Sami Aldalahmeh. This is an open access article distributed under the Creative Commons Attribution License, which permits unrestricted use, distribution, and reproduction in any medium, provided the original work is properly cited.

\begin{abstract}
In this paper, we propose a solution to find the angle of arrival (AOA), delay, and the complex propagation factor for the monostatic multiple-input multiple-output (MIMO) radar system. In contrast to conventional iterative computationally demanding estimation schemes, we propose a closed form solution for most of the previous parameters. The solution is based on forming an approximate correlation matrix of the received signals at the MIMO radar receiver end. Then, an eigenvalue decomposition (EVD) is performed on the formed approximate correlation matrix. The AOAs of the received signals are deduced from the corresponding eigenvectors. Then, the delays are estimated from the received signal matrix properties. This is followed by forming structured matrices which will be used to find the complex propagation factors. These estimates can be used as initializations for other MIMO radar methods, such as the maximum likelihood algorithm. Simulation results show significantly low root mean square error (RMSE) for AOAs and complex propagation factors. On the other hand, our proposed method achieves zero RMSE in estimating the delays for relatively low signal-to-noise ratios (SNRs).
\end{abstract}

\section{Introduction}

Multiple-input multiple-output (MIMO) radar is becoming increasingly popular due to its ability to overcome the fluctuation in the received power caused by varying radar cross section [1]. Thus, it is proposed in the literature as a tool to estimate the location of different targets [2-8]. Radar signals are transmitted from multiple transmitter antennas; then, they are reflected off targets and received back at the radar, hence providing the required receiver diversity. However, this operation requires estimating the angle of arrivals (AOAs) and delays of the received signals, which is usually done via a maximum likelihood (ML) method [9]. Nonetheless, the ML is computationally expensive because of its iterative nature. Worse yet, it might converge to a local minima leading to erroneous estimated parameters, providing inappropriate initial values fed to the $\mathrm{ML}$ algorithm. As a result, a need rises to provide a closed form solution to the estimation problem, which might also serve as an appropriate initial guess for the ML.
Several articles in the literature proposed direction finding methods for the bistatic MIMO radar system where the transmitter and receiver are located in different positions such as in [5]. On the other hand, many MIMO radar models were proposed in the literature such as in [6], which utilize the monostatic MIMO radar model where the transmitter and receiver are colocated. In this paper, we utilize the monostatic MIMO radar model in [6] with some extensions to fit the proposed solution. The proposed method starts by computing an approximate correlation matrix upon which we apply eigenvalue decomposition (EVD) to find the AOAs from the corresponding eigenvectors. However, it is noticed that when the received signal is stacked in a matrix form, a specific number of columns at the beginning of the matrix should be ideally zeros. The number of these columns is related to the delay of the received signal reflected off the nearest target. This property is used to independently estimate the delays. Then, the estimated AOAs and delays are used to form structured matrices which are utilized to find the complex propagation factors for each target. These 
estimates can be used as initializations for other adaptive MIMO radar estimating methods as in [6].

The rest of the paper is organized as follows: Section 2 presents the problem formulation part, Section 3 contains the proposed solution, and Section 4 shows simulation results. Finally, we conclude in Section 5.

\section{Problem Formulation}

In this section, we present the system model for the monostatic MIMO radar which is similar to the one used in [6]. Assume that the radar consists of colocated $N_{\mathrm{T} x}$ and $N_{\mathrm{R} x}$ transmitting and receiving uniform linear array (ULA) antennas, respectively. Let the transmitting antennas transmit a pulsed signal $a(t)$ that is modulated by the sequences $m_{i}(t)$ (for $i=1 \cdots$ $N_{\mathrm{T} x}$ ), having a period of $T_{b}$ and consisting of $N_{c}$ chips. These signals are uncorrelated over time, and antenna elements, i.e.,

$$
E\left[m_{i}(t) m_{j}(t-\tau)\right]= \begin{cases}0, & \text { if } i \neq j \text { or } \tau \neq 0 \\ 1, & \text { otherwise }\end{cases}
$$

where $E[\cdot]$ is the expectation operator and $\tau$ is some delay.

For $K$ targets, the manifolds of the transmitted and received signals are, respectively,

$$
\begin{aligned}
& \mathbf{s}_{\mathrm{T} x}\left(\theta_{k}\right)=\exp \left\{j\left[\begin{array}{lll}
\mathbf{r}_{x}, & \mathbf{r}_{y}, & \mathbf{r}_{z}
\end{array}\right]_{\mathrm{T}_{x}} \mathbf{k}\left(\theta_{k}\right)\right\} \quad\left(N_{\mathrm{T} x} \times 1\right), \\
& \mathbf{s}_{\mathrm{R} x}\left(\theta_{k}\right)=\exp \left\{-j\left[\begin{array}{lll}
\mathbf{r}_{x}, & \mathbf{r}_{y}, & \mathbf{r}_{z}
\end{array}\right]_{\mathrm{R}_{x}} \mathbf{k}\left(\theta_{k}\right)\right\} \quad\left(N_{\mathrm{R} x} \times 1\right), \\
& \mathbf{k}\left(\theta_{k}\right)=\frac{2 \pi f_{c}}{c}\left[\begin{array}{lll}
\cos \theta_{k}, & \sin \theta_{k}, \quad 0
\end{array}\right]^{T},
\end{aligned}
$$

where $(\cdot)^{T}$ is the transpose operator, $\left[\begin{array}{lll}\mathbf{r}_{x}, & \mathbf{r}_{y}, & \mathbf{r}_{z}\end{array}\right]_{\mathrm{T}_{x}}$ are the coordinates of transmitting antennas, $\left[\begin{array}{lll}\mathbf{r}_{x}, & \mathbf{r}_{y}, & \mathbf{r}_{z}\end{array}\right]_{\mathrm{R}_{x}}$ are the coordinates of receiving antennas, $f_{c}$ is the carrier frequency used, $c$ is the speed of light, and $\theta_{k}$ is the AOA of the $k$ th target (it is worth mentioning that we are considering the azimuth angle only in this paper).

Neglecting the clutter effect, the radar signal is reflected off the targets, hence experiencing different propagation factors and delays. Therefore, the received signal vector $\mathbf{x}(t)$ at time $t$ is represented as

$$
\mathbf{x}(t)=\sum_{k=1}^{K} \beta_{k} \mathbf{s}_{\mathrm{R} x}\left(\theta_{k}\right) \mathbf{s}_{\mathrm{T} x}^{H}\left(\theta_{k}\right) \mathbf{m}\left(t-\tau_{k}\right)+\mathbf{n}(t) \quad\left(N_{\mathrm{R} x} \times 1\right),
$$

where $(\cdot)^{H}$ is the Hermetian operator and

$$
\mathbf{m}(t)=\left[m_{1}(t), m_{2}(t), \cdots, m_{N_{\mathrm{T} x}}(t)\right]^{T} \quad\left(N_{\mathrm{T} x} \times 1\right)
$$

with $\beta_{k}$ is the complex propagation factor, which includes the path loss and reflection coefficient factor off the $k$ th target. Also, $\tau_{k}$ is the delay for the $k$ th target, $\mathbf{n}(t)$ is a $N_{\mathrm{R} x} \times 1$ complex Gaussian noise vector of zero mean, and $\sigma^{2}$ $\mathbf{I}_{N_{\mathrm{R} x} \times N_{\mathrm{R} x}}$ covariance matrix, with $\mathbf{I}_{N_{\mathrm{R} x} \times N_{\mathrm{R} x}}$ is $N_{\mathrm{R} x} \times N_{\mathrm{R} x}$ identity matrix. The radar system is shown in Figure 1.

Recall that the transmitted pulse has a total duration of $T_{b}$ and a chip duration of $T_{c}$, where $N_{c}=T_{b} / T_{c}$ chips per pulse. Then, the quantized delay is

$$
l_{k}=\left\lceil\frac{\tau_{k}}{T_{c}}\right\rceil_{\bmod \left\{N_{c}\right\}},
$$

where $\left\lceil\tau_{k} / T_{c}\right\rceil_{\bmod \left\{N_{c}\right\}}$ is the ceil of $N_{c}$ modulus of $\tau_{k} / T_{c}$.

Now, the received signal can be represented in a compact matrix form by stacking the discretized vectors as

$$
\mathbf{X}=\sum_{k=1}^{K} \beta_{k} \mathbf{s}_{\mathrm{R} x}\left(\theta_{k}\right)\left(\mathbf{s}_{\mathrm{T} x}\left(\theta_{k}\right)\right)^{H} \mathbf{M} \mathbf{J}^{l_{k}}+\mathbf{N} \quad\left(N_{\mathrm{R} x} \times L\right),
$$

where $\mathbf{M}$ is the matrix of stacked discretized $\mathbf{m}(t)$ vectors having a size of $N_{\mathrm{T} x} \times L$, where $L=N_{c}+Q$ is the number of pulses and $Q$ is the number of zero bins appended to $\mathbf{m}(t)$ which is the maximum tolerable delay. Whereas $\mathbf{J}$ is the discrete shifting matrix constructed as

$$
\mathbf{J}=\left[\begin{array}{cc}
\mathbf{0}_{L-1 \times 1}, & \mathbf{I}_{L-1 \times L-1} \\
0, & \mathbf{0}_{1 \times L-1}
\end{array}\right] \quad(L \times L)
$$

where $\mathbf{0}_{m \times n}$ is an all-zero $m$-by- $n$ matrix and $\mathbf{N}$ is an appropriate discrete $N_{\mathrm{R} x} \times L$ complex Gaussian noise matrix.

Equation (6) can be further simplified as follows:

$$
\mathbf{X}=\Gamma_{\mathrm{R} x} \boldsymbol{\beta} \Gamma_{\mathrm{T} x}^{H} \mathbf{\Upsilon} \boldsymbol{\Omega}+\mathbf{N}
$$

where $\Gamma_{\mathrm{R} x}$ is a $N_{\mathrm{R} x} \times K$ matrix with its $k$ th column contains $\mathbf{s}_{\mathrm{R} x}\left(\theta_{k}\right), \boldsymbol{\Gamma}_{\mathrm{T} x}$ is $N_{\mathrm{T} x} \times K$ matrix constructed in a similar manner from $\mathbf{s}_{\mathrm{T} x}\left(\theta_{k}\right), \boldsymbol{\beta}$ is a $K \times K$ diagonal matrix with its elements containing $\beta_{k}$, and

$$
\begin{aligned}
& \mathbf{Y}=[\mathbf{M}, \cdots, \mathbf{M}] \quad\left(N_{\mathrm{T} x} \times L K\right), \\
& \mathbf{\Omega}=\left[\mathbf{J}^{l_{1}}, \cdots, \mathbf{J}^{l_{K}}\right]^{T} \quad(L K \times L),
\end{aligned}
$$

where $\mathbf{Y}$ is a matrix with $\mathbf{M}$ is repeated $K$ times and $\boldsymbol{\Omega}$ is the universal shift matrix.

\section{The Proposed Method}

Our proposed method localizes the targets without using computationally demanding methods such as the ML. This is done by estimating the parameters of the first target, then subtracting its formed signal from the received signal in (6), and subsequently estimating the parameters of the second target and so on. The proposed method starts estimating the AOAs $\left(\theta_{k}\right.$ 's), then quantized time delays $\left(l_{k}\right.$ 's) and finally the complex propagation factors $\left(\beta_{k}\right.$ 's). 

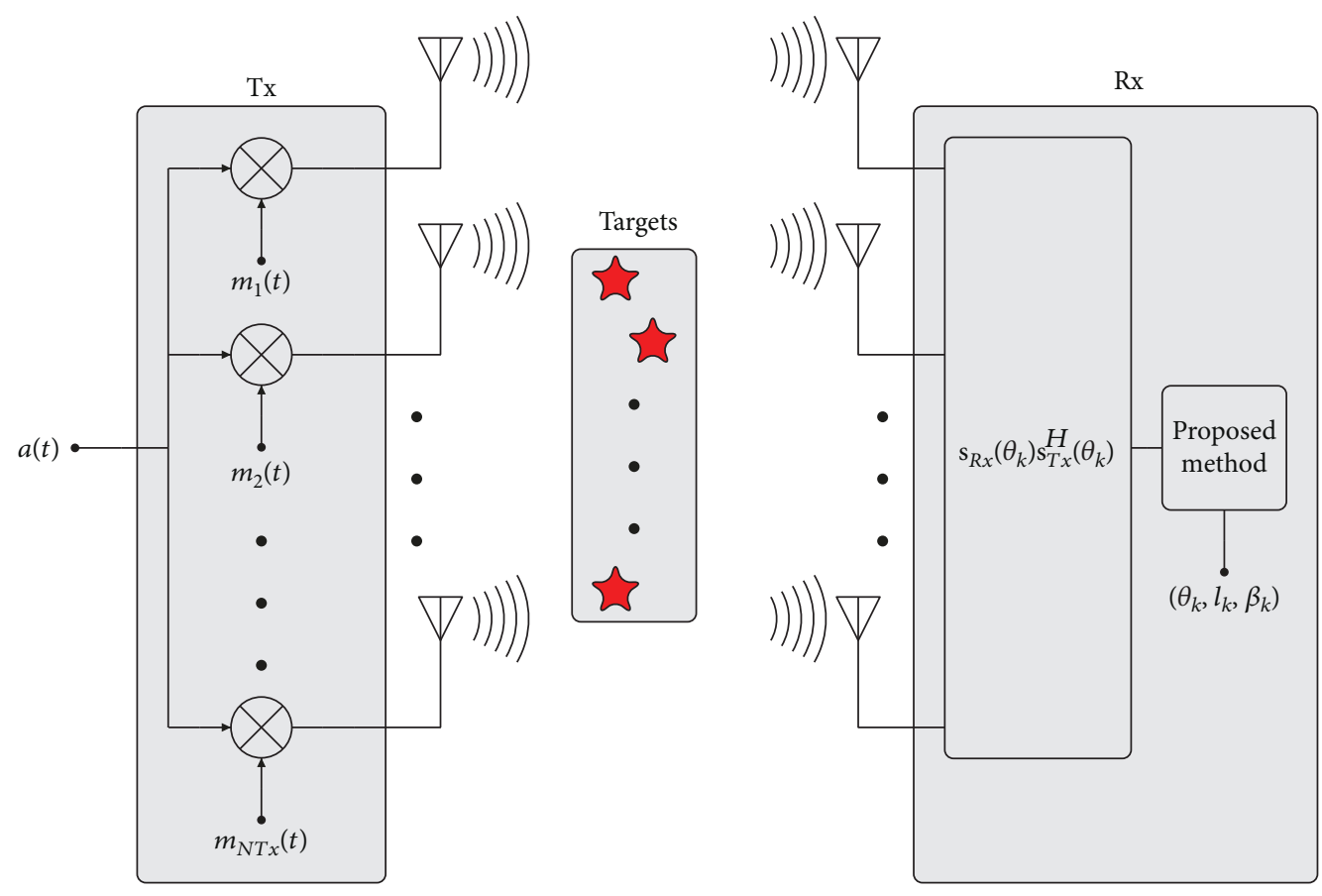

Figure 1: Monostatic radar system model.

3.1. AOA Estimation. Estimating the AOAs starts by forming the following approximate correlation matrix:

$$
\mathbf{R}=\mathbf{X X}^{H} \quad\left(N_{\mathrm{R} x} \times N_{\mathrm{R} x}\right) .
$$

From the definition of $\mathbf{X}$ in (8), it can be shown that

$$
\begin{aligned}
\mathbf{R} & =\boldsymbol{\Gamma}_{\mathrm{R} x} \boldsymbol{\Psi} \boldsymbol{\Gamma}_{\mathrm{R} x}^{H}+\sigma^{2} \mathbf{I}_{N_{\mathrm{R} x} \times N_{\mathrm{R} x}}, \\
\boldsymbol{\Psi} & =\boldsymbol{\beta} \boldsymbol{\Gamma}_{\mathrm{T} x}^{H} \boldsymbol{\Upsilon} \boldsymbol{\Omega}\left(\boldsymbol{\beta} \boldsymbol{\Gamma}_{\mathrm{T} x}^{H} \boldsymbol{\Upsilon} \boldsymbol{\Omega}\right)^{H} \\
& =\boldsymbol{\beta} \boldsymbol{\Gamma}_{\mathrm{T} x}^{H} \boldsymbol{\Upsilon} \boldsymbol{\Omega} \boldsymbol{\Omega}^{H} \boldsymbol{\Upsilon}^{H} \boldsymbol{\Gamma}_{\mathrm{T} x} \boldsymbol{\beta}^{H} .
\end{aligned}
$$

Next, let us denote $\mathbf{G}=\mathbf{\Upsilon} \boldsymbol{\Omega} \boldsymbol{\Omega}^{H} \mathbf{\Upsilon}^{H}$ which is a $N_{\mathrm{T} x} \times N_{\mathrm{T} x}$ matrix, then (12) becomes

$$
\boldsymbol{\Psi}=\boldsymbol{\beta} \boldsymbol{\Gamma}_{\mathrm{T} x}^{H} \mathbf{G} \boldsymbol{\Gamma}_{\mathrm{T} x} \boldsymbol{\beta}^{H}
$$

Now, from the assumption in (1) and assuming that $N_{c}$ is large enough, it can be shown that the matrix $\mathbf{G}$ is a diagonal matrix and its diagonal elements are $\left\{g_{0}, g_{1}\right.$, $\left.\cdots, g_{N_{\mathrm{Tx}}-1}\right\}$. These diagonal elements are positive-real and close in the value to each other. Similarly, denote the $K$ $\times K$ matrix $\mathbf{H}=\Gamma_{\mathrm{T} x}^{H} \mathbf{G} \boldsymbol{\Gamma}_{\mathrm{T} x}$. In this case and for a ULA antenna element system, it can be shown that the diagonal elements of $\mathbf{H}$ are

$$
h_{u, u}=\sum_{p=0}^{N_{\mathrm{Tx}}-1} g_{p}
$$

where $h_{u, u}$ is the $(u, u)$ element of the $\mathbf{H}$ matrix. Also, it can be shown that the off diagonal elements of $\mathbf{H}$ are given by

$$
h_{u, v}=\sum_{p=0}^{N_{\mathrm{T} x}-1} g_{p} e^{-j p w\left(\Delta_{x}\left(\cos \theta_{u}-\cos \theta_{v}\right)+\Delta_{y}\left(\sin \theta_{u}-\sin \theta_{v}\right)\right)} \quad \text { for } u \neq v,
$$

where $h_{u, v}$ is the $(u, v)$ element of the $\mathbf{H}$ matrix and $w=$ $2 \pi f_{c} / c$. Also, $\Delta_{x}$ and $\Delta_{y}$ are the distances between successive antenna elements on the $x$ and $y$ axes, respectively. Without loss of generality, the above can be simplified by setting $\Delta_{y}$ to zero yielding

$$
h_{u, v}=\sum_{p=0}^{N_{\mathrm{Tx}}-1} g_{p} e^{-j p w \Delta_{x}\left(\cos \theta_{u}-\cos \theta_{v}\right)} \quad \text { for } u \neq v .
$$

It is clear that $h_{u, v}$ is the sum of vectors of almost equal magnitudes and different phases. Thus, these off diagonal elements of $\mathbf{H}$ will sum up to values which are very small when compared to its diagonal elements (shown in (14)), i.e.,

$$
\left|h_{u, v}\right| \ll\left|h_{u, u}\right| \quad \text { for } u \neq v \text {. }
$$

We will call matrices satisfying the condition in (17) a quasidiagonal matrix. Quasidiagonal matrices retain the essential properties of diagonal matrices as it is shown in the simulation section (Section 4). So, $\mathbf{H}$ is a quasidiagonal matrix and so is $\boldsymbol{\Psi}$, since $\boldsymbol{\beta}$ is a diagonal matrix. 
The next step will be taking the EVD of $\mathbf{R}$ as follows:

$$
\mathbf{R}=\mathbf{U D U}^{H},
$$

where $\mathrm{U}$ is $N_{\mathrm{R} x} \times N_{\mathrm{R} x}$ size and its columns containing the eigenvectors of $\mathbf{R}$ and $\mathbf{D}$ is a $N_{\mathrm{R} x} \times N_{\mathrm{R} x}$ diagonal matrix containing the corresponding eigenvalues.

By comparing (11) and (18) and neglecting the noise term $\sigma^{2} \mathbf{I}_{N_{\mathrm{R} x} \times N_{\mathrm{R} x}}$ for the meanwhile, then,

$$
\Gamma_{\mathrm{R} x} \Psi \boldsymbol{\Gamma}_{\mathrm{R} x}^{H}=\mathbf{U D U}^{H} .
$$

So, it can be deduced that

$$
\Gamma_{\mathrm{R} x}=\mathrm{UT},
$$

where $\mathbf{T}$ is the appropriate matrix to change the basis vectors of size $N_{\mathrm{R} x} \times K$. Furthermore,

$$
\mathbf{D}=\mathbf{T} \Psi \mathbf{T}^{H} .
$$

It is already proven in [10] that since $\mathbf{D}$ is diagonal and $\Psi$ is quasidiagonal (which retain the properties of a diagonal matrix) and considering the first $K$ eigenvectors of $\mathbf{U}$, which correspond to the $K$ largest eigenvalues of $\mathbf{D}$, then we can deduce the AOAs of the $K$ targets $\left(\theta_{k}\right)$ implicitly as follows:

$$
\Delta_{x} \cos \left(\theta_{k}\right)+\Delta_{y} \sin \left(\theta_{k}\right)=\frac{\lambda \zeta_{k}}{2 \pi}
$$

where $\lambda$ is the wavelength of the transmitted signal. Now, by setting $\Delta_{y}=0$ (without loss of generality) to simplify the equation, we have

$$
\Delta_{x} \cos \left(\theta_{k}\right)=\frac{\lambda \zeta_{k}}{2 \pi}
$$

where

$$
\zeta_{k}=\frac{-\left(\sum_{\mu=1}^{N_{\mathrm{R}}-1} \angle\left(u_{\mu+1, k} / u_{\mu, k}\right)\right)}{N_{\mathrm{R} x}-1}
$$

and $u_{\mu, k}$ is the $\mu$ th element of the $k$ th eigenvector. More elaboration on how to find $\theta_{k}$ from (22) will be discussed in Section 4.

Algorithm 1 summarizes the phase estimation process for the targets.

Notice that $\{\hat{f}\}$ represents the estimate of some parameter $f$.

3.2. Time Delay and Complex Propagation Factor Estimation. In order to estimate the delays $\left(l_{k}\right.$ 's), we note that the received signal matrix $\mathbf{X}$ in (6) should have $l_{1}$ all-zero columns at the beginning of $\mathbf{X}$ in the noiseless case, which is the delay required for the signal reflected off the nearest target to reach the receiver. Hence, $l_{1}$ can be estimated by identifying the allzero columns from $\mathbf{X}$. However, because of the noise

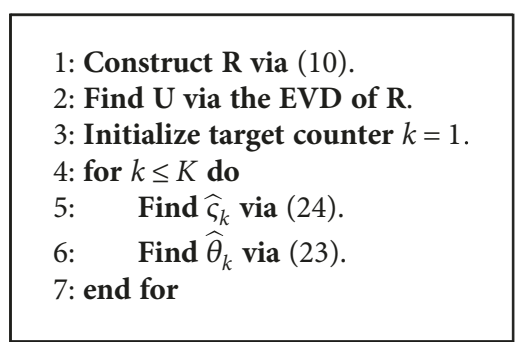

Algorithm 1: Estimating $\theta_{k}$ 's.



Algorithm 2: Estimating $l_{k}$.

presence, these values will not equal to zero exactly but will have relatively small magnitudes. So, to detect these values, we propose direct comparison with some threshold, which is chosen to be proportional to the magnitude average value of all elements of $\mathbf{X}$. The estimation process is summarized in Algorithm 2.

Note that $\bar{x}_{m}$ is the magnitude average of the $m$ th column elements of $\mathbf{X}$ and $\bar{x}$ is the magnitude average of all elements of $\mathbf{X}$. Also, $\gamma$ is an appropriately chosen threshold.

Next, we can compute $\beta_{k}$ for the $k$ th target (as proven in the Appendix and similar to what is shown in [8]), as follows:

$$
\widehat{\beta}_{k}=\frac{\left(\mathbf{s}_{\mathrm{R} x}\left(\widehat{\theta}_{k}\right)\right)^{H} \mathbf{X}\left(\widehat{\mathbf{J}}^{\widehat{l}_{k}}\right)^{H} \mathbf{M}^{H} \mathbf{s}_{\mathrm{T} x}\left(\widehat{\theta}_{k}\right)}{N_{\mathrm{R} x}\left(\mathbf{s}_{\mathrm{T} x}\left(\widehat{\theta}_{k}\right)\right)^{H} \mathbf{M} \widehat{\mathbf{J}}^{\widehat{l}_{k}}\left(\widehat{\mathbf{J}}^{\hat{l}_{k}}\right)^{H} \mathbf{M}^{H} \mathbf{s}_{\mathrm{T} x}\left(\widehat{\theta}_{k}\right)} .
$$

3.3. Localization Algorithm. Now, that we have the means to find all the required parameters $\widehat{\theta}_{k}, \widehat{l}_{k}$, and $\widehat{\beta}_{k}$, we can reformulate the received signal for a specific target as

$$
\widehat{\mathbf{X}}_{k}=\widehat{\beta}_{k} \mathbf{s}_{\mathrm{R} x}\left(\widehat{\theta}_{k}\right)\left(\mathbf{s}_{\mathrm{T} x}\left(\widehat{\theta}_{k}\right)\right)^{H} \mathbf{M} \widehat{\mathbf{J}}^{\widehat{l}_{k}} .
$$

As stated earlier, the estimated signals are subtracted from $\mathbf{X}$ in (6) in order to improve the $(k+1)$ th estimation in a successive manner. The complete localization algorithm is presented in Algorithm 3 (note that the outer loop in the algorithm refines the parameter estimation process). 


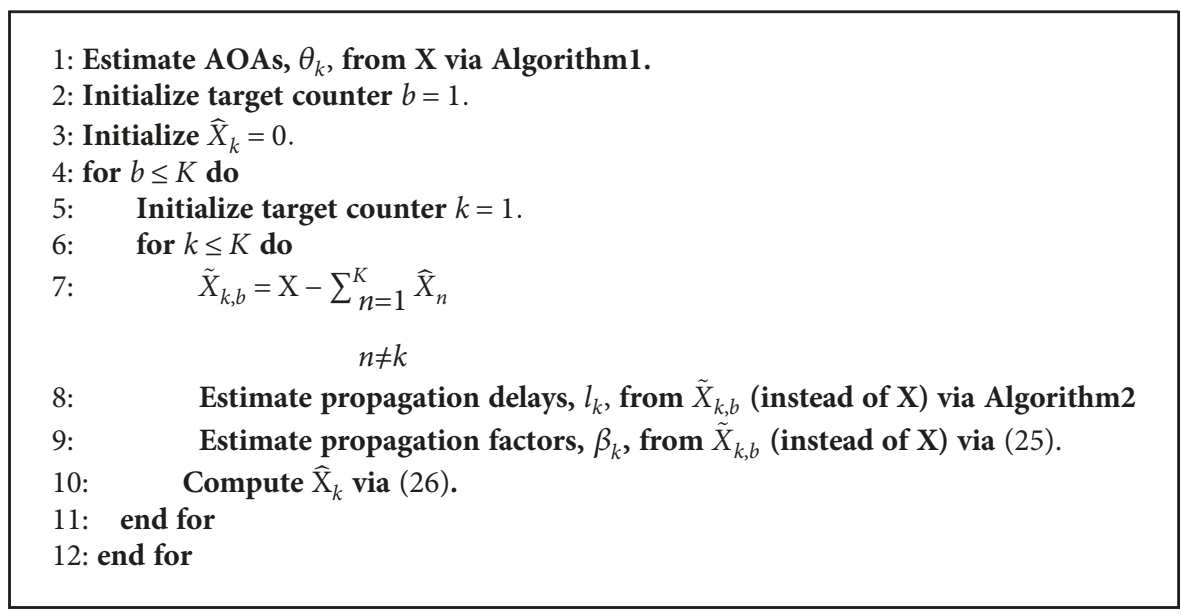

Algorithm 3: Localization algorithm.

\section{Simulation}

Simulations are performed to assess the performance of the proposed method. Two targets are assumed, i.e., $k=2$ with $\theta_{1}=30^{\circ}$ and $\theta_{2}=70^{\circ}$. Also, the complex propagation factors are assumed to be $\beta_{1}=0.7+0.7 j$ and $\beta_{2}=0.5-$ $0.25 \mathrm{j}$. The delays are assumed $l_{1}=4$ chips and $l_{2}=9$ chips with $N_{T c}=127$ and $Q=25$. The nonzero part of the message signal matrix $(\mathbf{M})$ is formed using Gold sequences. The results are averaged over 1000 ensembles. The number of antenna elements at the transmitter and receiver sides are $N_{\mathrm{T} x}=7$ and $N_{\mathrm{R} x}=9$, respectively. The threshold $\gamma$ is assumed to be 0.4 . Also, $\Delta_{x}=\lambda / 2$ and $\Delta_{y}=0$. So, (22) is simplified to

$$
\theta_{k}=\cos ^{-1}\left(\frac{\zeta_{k}}{\pi}\right) .
$$

The number of iterations of the outer loop in Algorithm 3 is two.

Figures 2, 3, and 4 show the root mean squared error (RMSE) of the AOAs, delays, and complex propagation factor magnitude estimation versus different signal-to-noise ratios (SNRs). It is clear from Figure 2 that the performance of AOA estimation for both targets is enhanced as the SNR increases up to some value where the performance plateaus due to the cross-correlation between different target signals (remember that Gold sequences have some small crosscorrelation values). Interestingly, Figure 3 shows that the delay RMSE reaches zero at relatively low SNRs. Whereas Figure 4 clearly shows that the RMSE of the complex propagation factor magnitude decreases for both targets as the SNR increases, as expected.

\section{Conclusion}

In this paper, we propose a computationally inexpensive algorithm to find the AOAs, delays, and complex propagation factors for monostatic MIMO radar systems. Most of

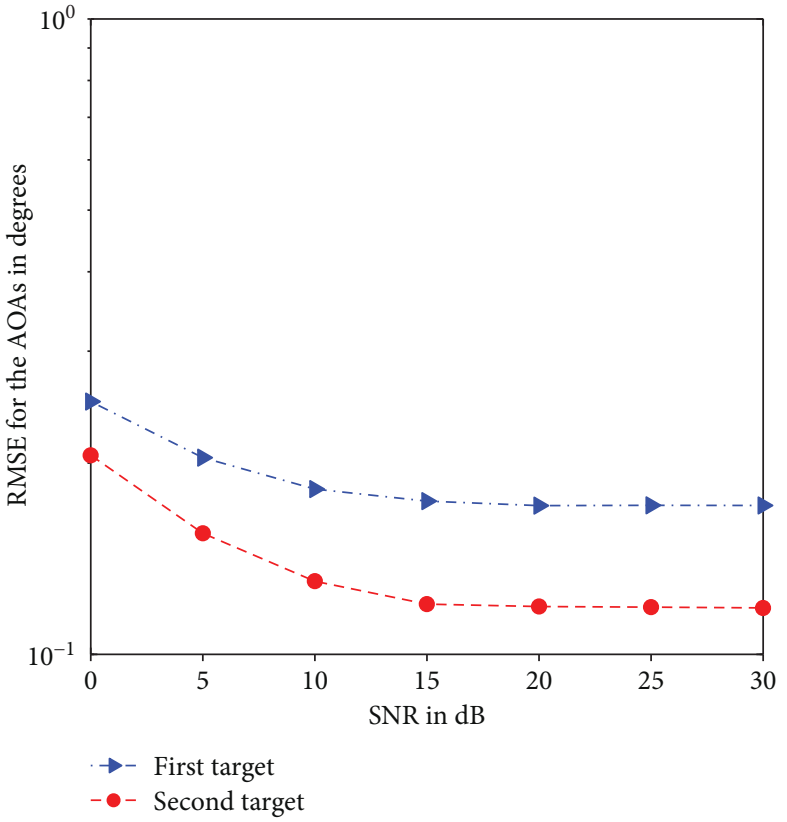

FIGURE 2: Root mean square error for the AOA in degrees vs. SNR in $\mathrm{dB}$ for $\gamma=0.4$.

the parameters are found in a closed-form manner. The algorithm starts by computing an approximate correlation matrix, then applying EVD to find the AOAs from the corresponding eigenvectors. The delay is estimated from the zero submatrix in the received signal stack. Then, we utilize the structured matrices from the estimated AOAs and delays to find the complex propagation factors for each target. These estimates can be used as initializations for other iterative MIMO radar methods. Simulation results, on one hand, show relatively low RMSE for the AOAs and complex propagation factors. And on the other hand, simulation results show virtually zero RMSE for the delay estimation. 




FIGURe 3: Root mean square error for the delay in chip time $T_{c}$ vs. SNR in $\mathrm{dB}$ for $\gamma=0.4$.

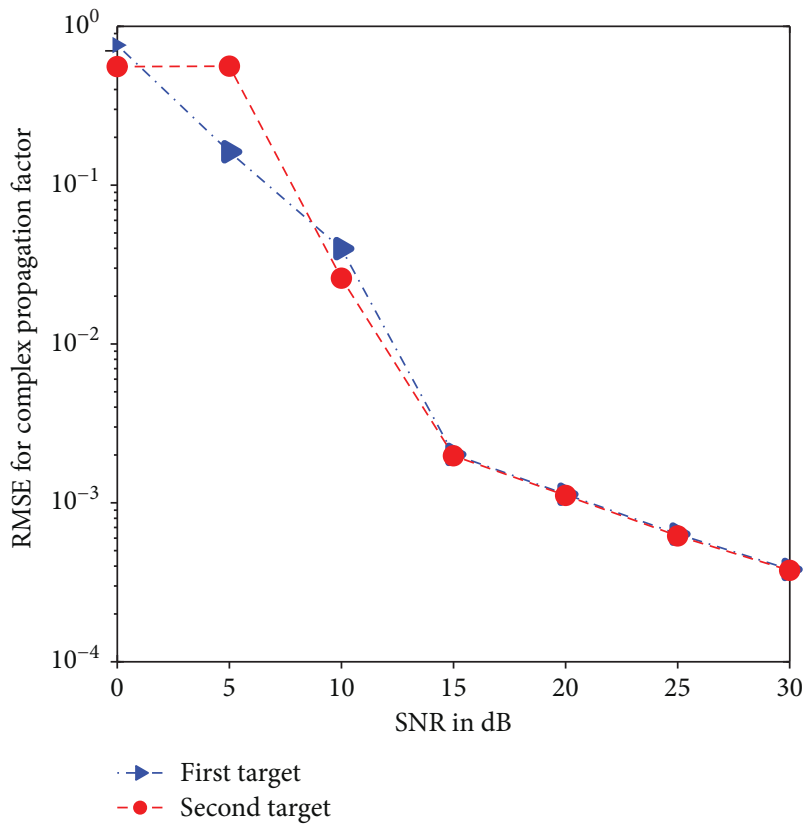

FIGURE 4: Root mean square error for the complex propagation factor magnitude vs. SNR in $\mathrm{dB}$ for $\gamma=0.4$.

\section{Appendix}

In this section, we will prove the equation in (25). To do so, let us minimize the mean squared error (MSE) of the received signal $\mathbf{X}$ in (6) with respect to $\beta_{k}$, i.e.,

$$
\min _{\beta_{k}}\left\|\mathbf{X}-\sum_{k=1}^{K} \beta_{k} \mathbf{s}_{\mathrm{R} x}\left(\theta_{k}\right)\left(\mathbf{s}_{\mathrm{T} x}\left(\theta_{k}\right)\right)^{H} \mathbf{M} \mathbf{J}^{l_{k}}\right\|^{2},
$$

which can be written in the form

$$
\begin{aligned}
\min _{\beta_{k}}\left[\mathbf{X}-\sum_{k=1}^{K} \beta_{k} \mathbf{s}_{\mathrm{R} x}\left(\theta_{k}\right)\left(\mathbf{s}_{\mathrm{T} x}\left(\theta_{k}\right)\right)^{H} \mathbf{M} \mathbf{J}^{l_{k}}\right] \\
\cdot\left[\mathbf{X}-\sum_{k=1}^{K} \beta_{k} \mathbf{s}_{\mathrm{R} x}\left(\theta_{\mathrm{k}}\right)\left(\mathbf{s}_{\mathrm{T} x}\left(\theta_{k}\right)\right)^{H} \mathbf{M} \mathbf{J}^{l_{k}}\right]^{H} .
\end{aligned}
$$

Now, take the derivative of (A.2) with respect to $\beta_{k}$ and equate it to zero as follows:

$$
\begin{aligned}
2 \beta_{k} \mathbf{s}_{\mathrm{R} x}\left(\theta_{k}\right)\left(\mathbf{s}_{\mathrm{T} x}\left(\theta_{k}\right)\right)^{H} \mathbf{M} \mathbf{J}^{l_{k}}\left(\mathbf{J}^{l_{k}}\right)^{H} \mathbf{M}^{H} \mathbf{s}_{\mathrm{T} x}\left(\theta_{k}\right) \\
\cdot\left(\mathbf{s}_{\mathrm{R} x}\left(\theta_{k}\right)\right)^{H}-2 \mathbf{X}\left(\mathbf{J}^{l_{k}}\right)^{H} \mathbf{M}^{H} \mathbf{s}_{\mathrm{T} x}\left(\theta_{k}\right)\left(\mathbf{s}_{\mathrm{R} x}\left(\theta_{k}\right)\right)^{H}=0 .
\end{aligned}
$$

Now, dividing both sides by 2 and multiplying the left side and right side of (A.3) by $\left(\mathbf{s}_{\mathrm{R} x}\left(\theta_{k}\right)\right)^{H}$ and $\mathbf{s}_{\mathrm{R} x}\left(\theta_{k}\right)$, respectively, lead to

$$
\begin{aligned}
\beta_{k}\left(\mathbf{s}_{\mathrm{R} x}\left(\theta_{k}\right)\right)^{H} \mathbf{s}_{\mathrm{R} x}\left(\theta_{k}\right)\left(\mathbf{s}_{\mathrm{T} x}\left(\theta_{k}\right)\right)^{H} \mathbf{M} \mathbf{J}^{l_{k}}\left(\mathbf{J}^{l_{k}}\right)^{H} \mathbf{M}^{H} \mathbf{s}_{\mathrm{T} x}\left(\theta_{k}\right) \\
\cdot\left(\mathbf{s}_{\mathrm{R} x}\left(\theta_{k}\right)\right)^{H} \mathbf{s}_{\mathrm{R} x}\left(\theta_{k}\right)-\left(\mathbf{s}_{\mathrm{R} x}\left(\theta_{k}\right)\right)^{H} \mathbf{X}\left(\mathbf{J}^{l_{k}}\right)^{H} \mathbf{M}^{H} \mathbf{s}_{\mathrm{T} x}\left(\theta_{k}\right) \\
\cdot\left(\mathbf{s}_{\mathrm{R} x}\left(\theta_{k}\right)\right)^{H} \mathbf{s}_{\mathrm{R} x}\left(\theta_{k}\right)=0 .
\end{aligned}
$$

But, $\left(\mathbf{s}_{\mathrm{R} x}\left(\theta_{k}\right)\right)^{H} \mathbf{s}_{\mathrm{R} x}\left(\theta_{k}\right)=N_{\mathrm{R} x}$. So,

$$
\begin{aligned}
\beta_{k} N_{\mathrm{R} x}^{2}\left(\mathbf{s}_{\mathrm{T} x}\left(\theta_{k}\right)\right)^{H} \mathbf{M} \mathbf{J}^{l_{k}}\left(\mathbf{J}^{l_{k}}\right)^{H} \mathbf{M}^{H} \mathbf{s}_{\mathrm{T} x}\left(\theta_{k}\right) \\
\quad-N_{\mathrm{R} x}\left(\mathbf{s}_{\mathrm{R} x}\left(\theta_{k}\right)\right)^{H} \mathbf{X}\left(\mathbf{J}^{l_{k}}\right)^{H} \mathbf{M}^{H} \mathbf{s}_{\mathrm{T} x}\left(\theta_{k}\right)=0 .
\end{aligned}
$$

Dividing both sides of (A.5) by $N_{\mathrm{R} x}$, then,

$$
\begin{aligned}
\beta_{k} N_{\mathrm{R} x}\left(\mathbf{s}_{\mathrm{T} x}\left(\theta_{k}\right)\right)^{H} \mathbf{M} \mathbf{J}^{l_{k}}\left(\mathbf{J}^{l_{k}}\right)^{H} \mathbf{M}^{H} \mathbf{s}_{\mathrm{T} x}\left(\theta_{k}\right) \\
-\left(\mathbf{s}_{\mathrm{R} x}\left(\theta_{k}\right)\right)^{H} \mathbf{X}\left(\mathbf{J}^{l_{k}}\right)^{H} \mathbf{M}^{H} \mathbf{s}_{\mathrm{T} x}\left(\theta_{k}\right)=0 .
\end{aligned}
$$

Next, rearranging (A.6) leads to

$$
\beta_{k}=\frac{\left(\mathbf{s}_{\mathrm{R} x}\left(\theta_{k}\right)\right)^{H} \mathbf{X}\left(\mathbf{J}^{\mathbf{J}_{k}}\right)^{H} \mathbf{M}^{H} \mathbf{s}_{\mathrm{T} x}\left(\theta_{k}\right)}{N_{\mathrm{R} x}\left(\mathbf{s}_{\mathrm{T} x}\left(\theta_{k}\right)\right)^{H} \mathbf{M} \mathbf{J}^{\mathbf{l}_{k}}\left(\mathbf{J}^{l_{k}}\right)^{H} \mathbf{M}^{H} \mathbf{s}_{\mathrm{T} x}\left(\theta_{k}\right)} .
$$

So, (25) is proven.

\section{Data Availability}

The data is available through simulation of the derived equations in the paper. 


\section{Conflicts of Interest}

The authors declare that there is no conflict of interest regarding the publication of this paper.

\section{References}

[1] E. Fishler, A. Haimovich, R. Blum, D. Chizhik, L. Cimini, and R. Valenzuela, "MIMO radar: an idea whose time has come," in Proceedings of the 2004 IEEE Radar Conference (IEEE Cat. No.04CH37509), pp. 71-78, Philadelphia, PA, USA, April 2004.

[2] P. Stoica, J. Li, and Y. Xie, "On probing signal design for MIMO radar," IEEE Transactions on Signal Processing, vol. 55, no. 8, pp. 4151-4161, 2007.

[3] K. Gao, H. Shao, J. Cai, H. Chen, and W.-Q. Wang, "Frequency diverse array MIMO radar adaptive beamforming with rangedependent interference suppression in target localization," International Journal of Antennas and Propagation, vol. 2015, Article ID 358582, 10 pages, 2015.

[4] X. Li, D. Wang, and X. Ma, “Three-dimensional target localization and Cramér-Rao bound for two-dimensional OFDMMIMO radar," International Journal of Antennas and Propagation, vol. 2017, Article ID 4171452, 14 pages, 2017.

[5] C. Yunhe, Z. Zijing, W. Shenghua, and D. Fengzhou, "Direction finding for bistatic MIMO radar with uniform circular array," International Journal of Antennas and Propagation, vol. 2013, Article ID 674878, 6 pages, 2013.

[6] K. Luo and A. Manikas, "Joint transmitter-receiver optimization in multitarget MIMO radar," IEEE Transactions on Signal Processing, vol. 65, no. 23, pp. 6292-6302, 2017.

[7] L. Xu, J. Li, and P. Stoica, "Radar imaging via adaptive MIMO techniques," in 2006 14th European Signal Processing Conference, pp. 1-5, Florence, Italy, September 2006.

[8] K. Luo and A. Manikas, "Superresolution multitarget parameter estimation in MIMO radar," IEEE Transactions on Geoscience and Remote Sensing, vol. 51, no. 6, pp. 3683-3693, 2013.

[9] B. Tang, J. Tang, Y. Zhang, and Z. Zheng, "Maximum likelihood estimation of DOD and DOA for bistatic MIMO radar," Signal Processing, vol. 93, no. 5, pp. 1349-1357, 2013.

[10] S. O. Al-Jazzar, A. Muchkaev, A. Al-Nimrat, and M. Smadi, "Low complexity and high accuracy angle of arrival estimation using eigenvalue decomposition with extension to $2 \mathrm{D}$ AOA and power estimation," EURASIP Journal on Wireless Communications and Networking, vol. 2011, no. 1, 2011. 


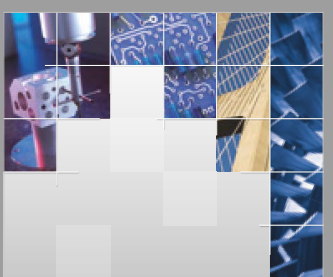

\section{Enfincering}
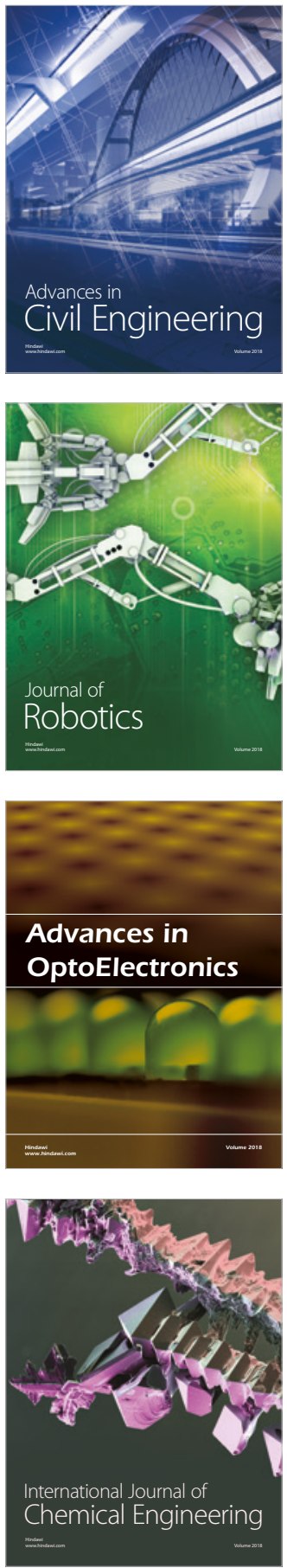

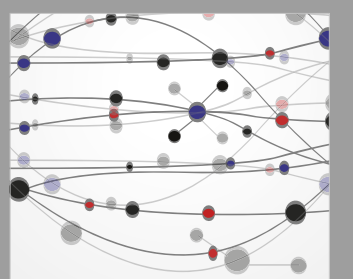

\section{Rotating \\ Machinery}

The Scientific World Journal

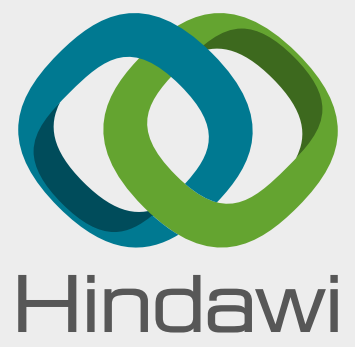

Submit your manuscripts at

www.hindawi.com



\section{Advances \\ Multimedia}
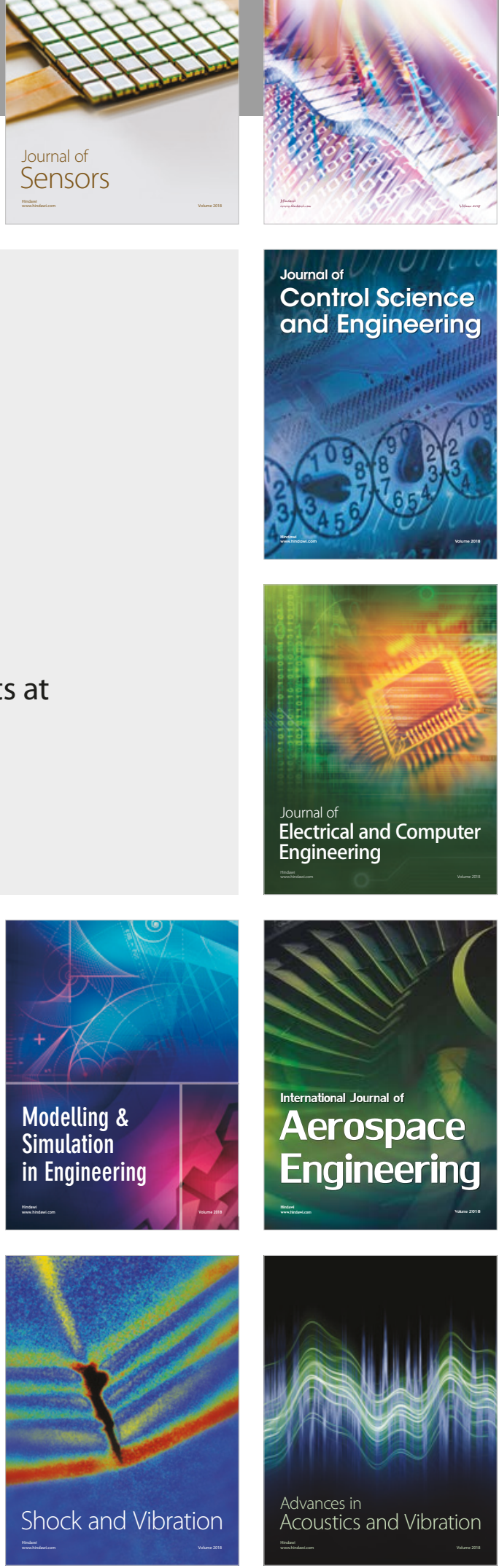